\title{
Gamificação Aplicada à Gestão do Conhecimento em Projetos: Um Mapeamento Sistemático
}

Pedro Henrique Dias Valle, Universidade de São Paulo, pedrohenriquevalle@usp.br Maria do Carmo Assis Todorov, Centro Universitário FMU, madu4@ hotmail.com

Resumo: O conhecimento gerado nas diversas organizações é visto como um importante recurso que pode ser utilizado no gerenciamento de projetos. Porém, registrar esse conhecimento é algo muito difícil, já que diversos fatores podem ocorrer ao longo da execução de projetos nas organizações. Nesse contexto, as organizações precisam de mecanismos para integrar e registrar os conhecimentos que são gerados nos projetos das organizações por meio do compartilhamento do conhecimento que é gerado entre os indivíduos elou por meio do armazenamento desses conhecimentos em documentos ou sistemas de informação. Nessa perspectiva, a gamificação tem sido explorada como um mecanismo de apoio ao compartilhamento dos conhecimentos existentes para novos indivíduos. Nesse sentido, este trabalho teve como principal objetivo identificar abordagens que utilizam a gamificação como recurso de apoio a gestão do conhecimento. Para isso, um mapeamento sistemático foi conduzido utilizando guidelines bem conhecidas na literatura. Nesse trabalho foram considerados 15 estudos primários para coletar as seguintes informações: (i) quais elementos de gamificação são utilizados para apoiar a gestão do conhecimento; (ii) quais são os benefícios na utilização da gamificação na gestão do conhecimento; e (iii) quais os tipos de conhecimentos relevantes para as organizações no contexto de gamificação. Como resultado, obteve-se uma visão geral sobre como a gamificação tem apoiado a gestão de conhecimento em projetos.

Palavras-chave: Elementos de Jogos, Conhecimento, Gestão de Projetos.

\section{Gamification Applied in Knowledge Management in Projects: A Systematic Mapping}

\begin{abstract}
The knowledge generated in different organizations is considered an important resource to be used in project management. However, it is very difficult to register this knowledge since several factors can occur during the cycle life of projects in organizations. In this sense, organizations need mechanisms to integrate and register knowledge that is generated in organizations' projects through the sharing of knowledge that is generated among individuals and from storing that knowledge in documents or information systems. In this perspective, gamification has been explored as a mechanism to support the sharing of knowledge for new individuals. In this sense, the goal of this paper is to identify approaches that use gamification as a resource to support knowledge management. For this, we carried out a systematic mapping using guidelines well established in the literature. We selected 15 primary studies to obtain the following information: (i) which gamification elements are used to support knowledge management; (ii)which are the benefits of using gamification in knowledge management; and (iii) which types of knowledge are relevant to organizations are explored in gamification context. As a result, we obtained an overview of how gamification has supported project knowledge management.
\end{abstract}

Keywords: Games Elements, Knowledge, Project Management. 


\section{Introdução}

O gerenciamento de projetos é o processo de aplicação de conhecimentos, habilidades e técnicas para a condução de projetos de forma efetiva e eficaz (RINC, 2014). Esse gerenciamento gera uma base de conhecimento proveniente da experiência e análise dos especialistas e também a partir do sucesso e fracasso das atividades executadas (JANNUZZI; FALSARELLA; ROBERTA, 2016). O conhecimento gerado é conhecido por muitas organizações como lições aprendidas, sendo considerado um importante elemento no gerenciamento de projetos (CARVALHO; FERNANDES; ARAÚJO, 2016).

No entanto, registrar esse conhecimento é muito difícil, uma vez que esse registro é feito apenas no encerramento do projeto e novas ações que ocorrem durante a execução do projeto podem ser esquecidos nessa etapa (PESSOA; SILVA; NASSIF, 2015). Outro ponto que dificulta o registro do conhecimento é que muitos membros da equipe que participaram do projeto são alocados para os próximos projetos e, consequentemente, eles ficam desmotivadas para o encerramento do projeto atual. Além disso, registrar as lições aprendidas em um único momento do projeto pode não ser suficiente para registrar todo o conhecimento gerado (CARVALHO; FERNANDES; ARAÚJO, 2016).

Nesse sentido, muitas organizações têm implantado o gerenciamento do conhecimento em projetos. Quando esse gerenciamento é realizado de forma efetiva e planejada, pode-se contribuir positivamente para alcançar os objetivos da organização, sendo considerado um diferencial estratégico, uma vez que o mercado atual está cada vez mais competitivo e com diversos concorrentes (BENOSSI, 2009). A gestão do conhecimento é o processo de criação continuo de novos conhecimentos, nos quais são amplamente disseminados por meio das organizações e são incorporados em produtos, serviços, tecnologias e sistemas (TAKEUCHI; NONAKA, 2009).

A gestão do conhecimento está relacionada, principalmente, à otimização do valor econômico que é entregue (SHINODA, 2012). Portanto, organizações que são compostas por ambientes em constantes mudanças devem processar informações de forma eficiente e promover a criação do conhecimento. De acordo com Bayart et al. (2009) um dos principais desafios da gestão do conhecimento é a transformação do conhecimento tácito em conhecimento explícito (CUFFA et al. 2016). Outro desafio é garantir que os conhecimentos gerados por meio de cada projeto, não se percam após o encerramento dos projetos (SHINODA, 2012).

Apesar dos projetos serem únicos, muitos elementos comuns podem ser retomados a cada novo projeto, tais como: clientes, setores e a metodologia utilizada em projetos anteriores. Portanto, é importante que as organizações utilizem mecanismos para integrar e registrar os conhecimentos que foram gerados em projetos anteriores, seja disseminando o conhecimento gerado para outros indivíduos ou armazenando esses conhecimentos em documentos e sistemas de informação (SHINODA, 2012). Nessa perspectiva, a gamificação pode ser utilizada como um mecanismo de apoio à disseminação do conhecimento para novos indivíduos (KAPP, 2012).

A gamificação pode ser definida como a utilização de conceitos de jogos em outro contexto que não está relacionado a jogos (DETERDING et al. 2011). Existem diversos elementos de gamificação que podem ser utilizados para disseminação do conhecimento, tais como: narrativas, feedback, recompensas, conflitos, classificação, competição, objetivos 
claros, níveis, distintivos, diversão, interação, interatividade, entre outros.

No contexto de gestão do conhecimento, Rinc (2014) investigou a utilização de elementos de gamificação para incentivar práticas de gestão do conhecimento nas organizações. Araújo, Belato and Fontana (2018) propuseram às empresas a utilização da gamificação para estimular os funcionários a atingirem suas metas por meio da utilização de jogos empresariais e gamificação dos treinamentos, potencializando assim, a absorção do conhecimento proposto pela organização.

Apesar do crescente interesse de pesquisas em gestão do conhecimento, ainda existe uma carência de trabalhos que indiquem quais elementos de gamificação são mais adequados para a gestão do conhecimento em projetos, e também sobre como a gamificação deve ser projetada para alcançar resultados positivos no contexto da gestão do conhecimento em projetos. Portanto, o principal objetivo deste estudo é identificar como a gamificação tem sido utilizada para apoiar a gestão do conhecimento em projetos. Para isso, foram identificados: (i) os elementos de gamificação utilizados parar apoiar a gestão do conhecimento em projetos; (i) os principais benefícios na utilização da gamificação na gestão do conhecimento em projetos; e (iii) os tipos de conhecimento explorados no contexto da gamificação.

\section{Materiais e métodos}

Para alcançar o objetivo deste trabalho, conduziu-se um mapeamento sistemático para identificar iniciativas que utilizaram a gamificação como recurso de apoio a gestão do conhecimento em projetos. O mapeamento sistemático é um tipo de pesquisa muito utilizada quando há um cenário abrangente e que tem por objetivo reunir o máximo de informações disponíveis sobre uma determinada área do conhecimento (PETERSEN et al. 2015). Para condução deste mapeamento sistemático foram utilizadas as guidelines definidas por Petersen et al. (2015).

Esse trabalho foi dividido em cinco etapas, sendo elas: (i) definição das questões de pesquisa; (ii) busca pelos estudos primários; (iii) seleção dos estudos por meio dos títulos e resumos; (iv) seleção dos estudos por meio dos critérios de inclusão e exclusão; e (v) extração das informações e mapeamento dos estudos. O mapeamento sistemático contemplou um período delimitado, entre os anos de 2010 a 2020. Esse período foi estabelecido porque a gamificação é uma área muito recente, e provavelmente os estudos que são interessantes para este trabalhos foram publicados nos últimos anos.

\subsection{Questões de pesquisa}

Neste trabalho, definiram-se três questões de pesquisa, sendo elas:

- $\mathrm{RQ}_{1}$ : Quais elementos de gamificação têm sido utilizados para apoiar a gestão do conhecimento em projetos?

- $\mathrm{RQ}_{2}$ : Quais são os principais benefícios em utilizar a gamificação na gestão do conhecimento em projetos?

- $\mathrm{RQ}_{3}$ : Quais são os tipos de conhecimentos explorados no contexto da gamificação?

\subsection{String de busca}

A string de busca é um mecanismo utilizado pelas bases de dados para identificar possíveis estudos para um determinado contexto. Neste trabalho foi considerado a seguinte string de busca: 
("Gamification") AND ("Knowledge Management" OR “Knowledge Sharing” OR “KM”)

Essa é uma string genérica, pois para cada base de dados, ela foi adaptada de acordo com suas particularidades. Além disso, foram formuladas strings de busca alternativas, as quais foram compostas pelos sinônimos da string genérica.

\subsection{Estratégias de busca}

A estratégia de busca dos trabalhos primários consistiu em duas etapas. Na primeira etapa, utilizaram-se bases de dados para identificar os estudos primários para responder as questões de pesquisas propostas. Nesse contexto, foram consideradas as seguintes bases de dados: IEEE Xplore, Scopus, ACM Digital Library, ISI Web of Science e El Compendex. Na segunda etapa, foram realizadas buscas manuais no Google Acadêmico e nos anais das principais conferências da área de gestão de projetos, gestão do conhecimento e gamificação para identificar estudos relevantes.

\subsection{Critérios para inclusão e exclusão dos estudos}

Foram definidos critérios de inclusão para selecionar os estudos primários que foram analisados, sendo eles: (i) estudos que apresentam relatos de experiência, estudos de casos ou abordagens que utilizem a gamificação como recurso de apoio a gestão do conhecimento; e (ii) estudos que utilizam a gamificação para apoiar a gestão do conhecimento no contexto de gestão de projetos.

É importante ressaltar que as negações dos critérios apresentados anteriormente foram utilizadas para a exclusão dos estudos retornados pelas máquinas de busca. Além disso, outros critérios de exclusão foram utilizados para seleção dos estudos, sendo eles: (i) estudos publicados em outros idiomas diferente de inglês ou português; (ii) estudos que sejam relatórios técnicos ou que não estejam disponíveis; e (iii) estudos que não apresentam a gamificação como um recurso de apoio à gestão do conhecimento.

\subsection{Extração dos dados}

As informações obtidas para responder as questões de pesquisa foram extraídas a partir dos estudos primários selecionados. Foram utilizados os seguintes passos:

1. Utilização da string de busca para identificar estudos que demonstrem como a gamificação pode apoiar a gestão do conhecimento em projetos;

2. Busca manual nos principais anais de conferências das áreas de gestão de projetos, gestão do conhecimento e gamificação, além de utilizar o Google Acadêmico de forma complementar;

3. Leitura dos títulos, resumos e palavras-chaves dos estudos retornados e selecionar um conjunto de estudos candidatos para responder as questões de pesquisa;

4. Leitura detalhada dos estudos selecionados, e aplicação dos critérios de inclusão e exclusão;

5. Seleção do conjunto final de estudos;

6. Extração das informações para responder as questões de pesquisa.

\section{Resultados e discussão}

Para responder as três questões de pesquisa propostas neste mapeamento sistemático foram selecionados 15 estudos primários. O processo de seleção dos estudos começou com a 
aplicação da string de busca nas bases de dados, retornando um conjunto de 171 estudo primários. O processo de busca e seleção dos estudos foi realizado no período de 01 de outubro de 2019 a 31 de janeiro de 2020. A ferramenta Parsifa $\bigsqcup^{1}$ foi utilizada para apoiar todo o processo seleção e extração das informações do mapeamento sistemático.

Foram removidos 17 estudos duplicados, e portanto, consideram-se apenas 154 estudos para aplicação dos critérios de inclusão e exclusão do conjunto original de estudos retornados. Outros quatro estudos foram identificados por meio do Google Acadêmico e adicionados ao conjunto de estudos. Esses estudos não foram retornados utilizando a string de busca.

O próximo passo foi a leitura dos títulos, resumos e palavras-chave para a seleção dos estudos que seriam analisados. Como resultado, foram selecionados apenas 22 estudos primários. Por fim, foram lidos os textos completos desses 22 estudos, aplicando os critérios de inclusão e exclusão e analisando se eles poderiam responder as questões de pesquisa propostas. Como resultado, 15 estudos foram selecionados para responder as questões de pesquisa. A lista completa dos estudos considerados pode ser observada na Tabela 11. Os estudos estão ordenados de acordo com o ano de publicação.

Tabela 1. Lista dos estudos primários selecionados

\begin{tabular}{|c|c|c|}
\hline ID & Título & Ano \\
\hline S1 & erious fun-effects of gamification on $\mathrm{kn}$ & 2014 \\
\hline $\mathrm{S} 2$ & $\mathrm{~g}$ & \\
\hline S3 & e management & 2015 \\
\hline $\mathrm{S} 4$ & . & 2016 \\
\hline S5 & on in knowledge 1 & 2016 \\
\hline S6 & $\begin{array}{l}\text { HPE Service Manager to Improve IT Service Desks' Knowledge } \\
\text { on }\end{array}$ & 2017 \\
\hline S7 & $\begin{array}{l}\text { ation of an enterprise collaboration system increases knowledge } \\
\text { an affordance approach }\end{array}$ & 2017 \\
\hline $\mathrm{S} 8$ & Chan & 2017 \\
\hline $\mathrm{Sc}$ & Know & 2018 \\
\hline S10 & ts of gamifi- & 2019 \\
\hline S1 & $\begin{array}{l}\text { I rather share my knowledge: Applying gamification approach and nudge the- } \\
\text { ory to develop an incentive system }\end{array}$ & 2019 \\
\hline S12 & amification: A case study of the & 2019 \\
\hline S13 & the Context of Knowledg & 2019 \\
\hline $\mathrm{S} 1$ & $\begin{array}{l}\text { Model for the Improvement of Knowledge Management Processes Based on } \\
\text { the Use of Gamification Principles in Companies in the Software Sector }\end{array}$ & 2019 \\
\hline S15 & & 2020 \\
\hline
\end{tabular}

Os estudos selecionados foram publicados a partir do ano de 2014 até 2020. Isso pode ser explicado pelo fato que a gamificação é uma área muito nova, a qual vem despertando o interesse de diversos pesquisadores nos últimos anos. Um aumento no interesse na utilização da gamificação na gestão do conhecimento foi observado no ano de 2019. Isso

${ }^{1}$ https://parsif.al/ 
demonstra um forte interesse da comunidade em utilizar gamificação para apoiar a gestão do conhecimento, devido aos diversos benefícios que ela fornece, dentre eles a motivação e o engajamento na troca de conhecimento entre as pessoas.

Esses estudos foram disponibilizados em diferentes veículos de publicação (anais de conferências, capítulos de livros e periódicos). Ao analisar a fonte dos estudos, pode-se observar uma tendência de estudos nessa área serem publicados em periódicos e anais de conferências. Isso é importante, pois ambos veículos são rigorosos com relação a contribuição e relevância dos estudos para a comunidade científica.

\subsection{Elementos de gamificação}

A primeira questão de pesquisa está relacionada com os elementos de gamificação que têm sido utilizados para apoiar a gestão do conhecimento em projetos. É importante ressaltar que todos os estudos analisados utilizaram elementos de gamificação para apoiar a gestão do conhecimento em projetos. Neste mapeamento sistemático, dez elementos de gamificação (i.e., pontos, distintivos/medalhas, classificação/ranking, recompensas, níveis, feedback, desafio, progresso, objetivos claros e histórias) foram identificados, sendo que o elemento de gamificação pontos foi considerado em todos os trabalhos analisados. O elemento de gamificação histórias foi utilizado em apenas um estudo (S1).

A Tabela 2 apresenta uma visão geral sobre quais elementos de gamificação foram considerados pelos estudos analisados.

Tabela 2. Elementos de gamificação considerados

\begin{tabular}{llc}
\hline $\begin{array}{l}\text { Elementos de } \\
\text { Gamificação }\end{array}$ & \multicolumn{1}{c}{ Estudos Primários } & Qtd. \\
\hline Pontos & S1, S2, S3, S4, S5, S6, S7, S8, S9, S10, S11, S12, S13, S14, S15 & 15 \\
Distintivos & S1, S2, S3, S4, S5, S6, S7, S8, S9, S11, S12, S13, S14, S15 & 14 \\
Classificação & S1, S2, S3, S6, S7, S8, S11, S12, S13, S14, S15 & 11 \\
Recompensas & S1, S3, S4, S5, S6, S7, S8, S9, S11, S12, S15 & 11 \\
Níveis & S1, S2, S4, S7, S10, S11 & 6 \\
Feedback & S1, S4, S6, S15 & 4 \\
Desafio & S1, S2, S3, S15 & 4 \\
Progresso & S1, S6, S8 & 3 \\
Objetivos Claros & S1, S9 & 2 \\
Histórias & S1 & 1 \\
\hline
\end{tabular}

Após analisar a Tabela 2, observaram-se que os elementos de gamificação pontos, distintivos, classificação, recompensas e níveis são os principais elementos considerados na da gestão do conhecimento em projetos. Em particular, no estudo S1 os autores classificaram os elementos de acordo com sua complexidade e se eles podiam ser utilizados de forma singular ou apenas em tarefas em grupos. No estudo S2, os elementos de gamificação estão focados na troca de conhecimento entre os indivíduos, as equipes, as unidades organizacionais, e as organizações. No estudo S3, os funcionários recebiam o elemento pontos após eles concluírem as tarefas proposta. Em seguida, os funcionários podiam utilizar esses pontos para comprar outros elementos, como as recompensas.

No estudo $\mathrm{S} 4$, os funcionários utilizavam um sistema online que incorporava elementos de gamificação. O sistema era composto de duas partes: (i) um repositório de co- 
nhecimento online, no qual os funcionários poderiam fornecer conhecimentos importantes para a empresa; e (ii) um jogo de estratégia que motivava os funcionários a interagir com o conteúdo do repositório de conhecimento. Reforçando a importância da gamificação, no estudo S5 os autores comentaram que a utilização da gamificação no sistema considerado para o estudo de caso foi devido a necessidade da empresa e não por que a gamificação é considerada um tema que está em ascensão na comunidade cientifica. Portanto, isso demonstra que a gamificação tem sido requerida na prática para apoiar a gestão do conhecimento em projetos. Nessa mesma perspectiva, no estudo S7, os autores investigaram as possibilidades fornecidas pela gamificação para os funcionários utilizarem esse mecanismo para contribuir no compartilhamento de informações/conhecimento.

No estudo S6, os autores criaram uma solução gamificada (utilizando pontos, progresso, recompensas, distintivos, classificação e feedback) para motivar as centrais de serviço de TI para gerar, compartilhar e melhorar continuamente qualidade do conhecimento que é usado para ajudar a atingir as metas desejadas de atendimento e melhorar a satisfação dos clientes. No estudo S8, os autores realizaram uma discussão sobre a utilização da gamificação na gestão do conhecimento. Para isso, os autores conduziram uma revisão de literatura com o objetivo de identificar os elementos que podem ser utilizados no contexto de gestão do conhecimento.

Nessa mesma perspectiva, uma ferramenta gamificada foi desenvolvida no estudo S9 com o objetivo de envolver os funcionários da empresa nos processos de disseminação e troca de conhecimentos dentro da organização. No estudo S10, os autores fizeram uma revisão de literatura para identificar elementos de gamificação e, posteriormente, adicionaram esses elementos em uma metodologia que eles propuseram no contexto do ensino superior.

A gamificação na gestão do conhecimento também foi aplicada em um domínio especifico. O estudo S11 propôs uma abordagem no contexto de indústrias de petróleo, utilizando a gamificação para promover o compartilhamento do conhecimento entre os funcionários. No contexto das universidades, no estudo S12 os autores realizaram um estudo de caso, no Laboratório de Governo Eletrônico da Universidade da Indonésia, considerando os elementos de gamificação: pontos, classificação, distintivo e recompensas.

No estudo S13, os autores apresentaram os resultados preliminares de um projeto chamado $\mathrm{KMM}+\mathrm{G}$. Este projeto explora uma abordagem para melhorar os processos de gestão do conhecimento em empresas de desenvolvimento de software utilizando a gamificação. No estudo S14, os autores apresentaram um modelo adaptado às condições das organizações, permitindo a identificação, criação, transferência e avaliação do conhecimento usando elementos de gamificação (pontos, classificação e distintivos).

Finalmente, no estudo S15, os autores analisaram os efeitos da gamificação na motivação e no comportamento dos funcionários no compartilhamento de conhecimento. Além disso, os autores discutiram os benefícios e os riscos da implementação de cada elemento de gamificação (feedback, desafios, classificação, recompensas, distintivos e pontos) considerados na sua abordagem.

A partir da análise realizada, identificaram-se os principais elementos de gamificação reportados na literatura para apoiar a gestão do conhecimento em projetos. Essa informação é relevante para a indústria e para comunidade científica, pois ambas podem estabelecer o planejamento de novas abordagens gamificadas no contexto de gestão do conhecimento. 
Uma vez que essas informações trazem conhecimentos de projetos anteriores que utilizaram a gamificação como recurso de apoio a gestão do conhecimento.

\subsection{Benefícios da gamificação}

A segunda questão de pesquisa está relacionada com os benefícios observados na utilização da gamificação na gestão de conhecimento em projetos. A maior parte dos estudos apontaram benefícios na utilização da gamificação na gestão do conhecimento em projetos. Em especial, no estudo S1 os elementos de gamificação ajudaram a superar as barreiras encontradas na troca de conhecimento entre os funcionários. Essas barreiras podem estar relacionadas ao trabalho e também a troca de conhecimento. Dentre elas, destacam-se: a falta de confiança, valorização e encorajamento.

Nos estudos S2, S9, S10, S13 e S14 os autores relataram que adição de elementos de gamificação contribuiu na motivação dos funcionários para o compartilhamento de conhecimento entre os funcionários da organização. Nos estudos S3 e S15, os autores relataram que a utilização da gamificação na gestão do conhecimento contribuiu, principalmente, para a hierarquia das necessidades proposta por Maslow, no nível mais alto. Essa hierarquia é composta por cinco categorias de necessidades humanas, sendo elas: fisiológicas, segurança, afeto, estima e as de auto realização. Em resumo, de acordo com estudos S3 e S15, a gamificação contribuiu na autoestima e auto realização, satisfazendo as necessidades mais alta da pirâmide de Maslow.

No estudo S4, os autores afirmaram que a gamificação contribuiu para o interesse e a diversão dos funcionários no compartilhamento de conhecimento, além de desenvolver as habilidades de autonomia e motivação dos envolvidos. Portanto, a utilização dos elementos de gamificação contribuiu de forma significativa para que os funcionários interagissem e trocassem conhecimentos entre si. No estudo S5, os autores relataram que a gamificação pode ser usada para auxiliar na identificação dos diferentes tipos de funcionários da empresa, além de visualizar suas habilidades e criar requisitos para novas habilidades. Ela também pode mostrar a dinâmica de interações entre os funcionários e os conhecimentos que será compartilhado. No estudo S6 e S11, os autores relataram que a gamificação melhorou o desempenho dos funcionários nas organizações.

No estudo $\mathrm{S} 7$, os resultados confirmam a ideia de que a gamificação aumenta o compartilhamento de conhecimento e, consequentemente, aumenta o envolvimento dos funcionários no compartilhamento do conhecimento. No estudo S8, os autores discutiram que o uso da gamificação na gestão do conhecimento pode ir muito além dos aspectos motivacionais. Dentre os benefícios observados, a gamificação pode contribuir para apoiar a flexibilidade, facilitar a transparência e, portanto, melhorar a confiança, visualizar habilidades e competências, além de gerar requisitos para novas competências e promover um ambiente colaborativo entre os profissionais do conhecimento.

Por fim, no estudo S12, os autores relataram que a gamificação ajuda na competição entre os funcionários e não contribuiu para o compartilhamento do conhecimento no contexto do estudo. Portanto, esse foi o único estudo que não apresentou uma melhora efetiva na gestão do conhecimento utilizando a gamificação. Nesse sentindo, observaram-se diversas evidências na literatura sobre os benefícios de utilizar gamificação como recurso de apoio a gestão do conhecimento, principalmente, com relação aos aspectos motivacionais. 


\subsection{Tipos de conhecimento}

A terceira questão de pesquisa está relacionada com os tipos de conhecimentos explorados no contexto da gamificação. Este estudo considerou os oito tipos de conhecimento relevantes para as organizações, conforme relatado por Shinoda (2012). Esses conhecimentos são considerados importantes para serem gerenciados em projetos. Os tipos de conhecimentos considerados foram: técnico, consultoria, processual: fases do projeto, processual: fases do gerenciamento de projetos, cliente: servidor, organização e contexto, quem sabe o que (know who), histórico de projetos e cultural.

Os estudos primários foram analisados visando identificar os tipos de conhecimentos considerados relevantes para as organizações que foram apoiados pela gamificação. A Tabela 3 sumariza as evidências obtidas para essa questão de pesquisa.

Tabela 3. Tipos de conhecimento considerados na gestão do conhecimento

\begin{tabular}{llc}
\hline Tipo de Conhecimento & Estudos Primários & Qtd. \\
\hline Cliente: servidor, organização e contato & S2, S7, S13, S14, S15 & 5 \\
Técnico & S1, S8, S10, S12, S14 & 5 \\
Consultoria & S5, S7, S10, S12 & 4 \\
Cultural & S3, S8, S9 & 3 \\
Quem sabe & S4, S11, S12 & 3 \\
Processual: fases do projeto & S6 & 1 \\
Histórico de projetos & S4 & 1 \\
Processual: fases do gerenciamento de projeto & S11 & 1 \\
\hline
\end{tabular}

Como pode ser observado na Tabela 3 , os conhecimentos de Cliente, Técnico e Consultoria foram considerados com maior frequência nos estudos que relataram a utilização da gamificação como recurso de apoio a gestão do conhecimento. Esses tipos de conhecimento estão relacionados principalmente aos clientes, projetos e negociação.

\section{Conclusões}

Este trabalho teve como principal objetivo identificar abordagens que utilizaram a gamificação para apoiar a gestão do conhecimento em projetos. Foram identificados 10 elementos de gamificação para apoiar a gestão do conhecimento, nos quais pontos, distintivos, classificação e recompensas foram os elementos mais utilizados pelas estudos analisados. Os estudos primários relataram diversos benefícios com a aplicação da gamificação na gestão do conhecimento em projetos, dentre eles destacam-se: (i) o aumento da motivação dos funcionários para troca e disseminação de informações/conhecimento entre as pessoas da organização; (ii) a melhora do desempenho dos funcionários na organização; e (iii) obtenção do último nível da hierarquia de Maslow que está relacionada com aspectos de autoestima e auto realização pelos funcionários.

Diferentes tipos de conhecimentos considerados relevantes para as organizações foram identificados, como aqueles relacionamentos principalmente aos clientes, projetos e negócios das organizações. Como trabalhos futuros, pretende-se desenvolver uma tipologia sobre elementos de gamificação para apoiar a gestão de conhecimento em projetos e avaliá-la em um cenário real com especialistas em gamificação e gestão do conhecimento. 


\section{Agradecimentos}

Os autores gostariam de agradecer ao Pecege, a CAPES - Código de Financiamento 001, e ao $\mathrm{CNPq}$ pelo apoio financeiro.

\section{Referências}

ARAúJO, V. R. d.; BELATO, M. H. V.; FONTANA, V. S. Gamificação como recurso inovador no processo de gestão do conhecimento e na tomada de decisão. In: Faculdade Multivix Cachoeiro de Itapemirim, 2018.

BAYART, C. et al. Serious games: leverage for knowledge management. The TQM Journal, v. 26, n. 3, p. 235-252, 2009.

BENOSSI, G. Gestão do conhecimento no treinamento e desenvolvimento de pessoas. Dissertação de Mestrado. Universidade de Campinas, v. 1, n. 1, p. 1-123, 2009.

CARVALHO, R. F. D. S.; FERNANDES, H. R. D. C.; ARAÚJO, E. A. D. A gestão do conhecimento para melhoria do gerenciamento de projetos. Ciências Sociais Aplicadas em Revista, v. 16, n. 31, p. 80-89, 2016.

CUFFA, D. de et al. O impacto da governança corporativa eletrônica na gestão do conhecimento. NAVUS-Revista de Gestão e Tecnologia, Serviço Nacional de Aprendizagem Comercial, v. 6, n. 4, p. 98-107, 2016.

DETERDING, S. et al. Gamification. using game-design elements in non-gaming contexts. In: Human Factors in Computing Systems (CHI). New York, NY, USA: Association for Computing Machinery, 2011.

JANNUZZI, C. S. C.; FALSARELLA, O. M.; ROBERTA, C. Gestão do conhecimento: um estudo de modelos e sua relação com a inovação nas organizações. Perspectivas em Ciência da Informação, v. 21, n. 1, p. 97-118, 2016.

KAPP, K. The Gamification of Learning and Instruction: Game-based Methods and Strategies for Training and Education: Wiley, 2012. (Pfeiffer essential resources for training and HR professionals). ISBN 9781118096345.

PESSOA, C. R. M.; SILVA, F. B.; NASSIF, M. E. The Relationships between Project Management and Knowledge Management: Where We Can Find Project Knowledge Management in the Project Management Process. Hershey, PA: IGI Global: In Handbook of Research on Effective Project Management through the Integration of Knowledge and Innovation, 2015.

PETERSEN, K. et al. Systematic mapping studies in software engineering. In: 12th International Conference on Evaluation and Assessment in Software Engineering (EASE) 12, 2015. p. $1-10$.

RINC, S. Integrating gamification with knowledge management. In: International Conference Management on Knowledge and Learning: International School for Social and Business Studies, 2014.

SHINODA, A. C. M. Gestão do conhecimento em projetos: um estudo sobre conhecimentos relevantes, fatores influenciadores e práticas em organizações projetizadas. Dissertação (Mestrado) — Universidade de São Paulo, 2012.

TAKEUCHI, H.; NONAKA, I. Gestão do conhecimento: Bookman Editora, 2009. 